\title{
Collapse of Small-Scale Density Perturbations during Preheating in Single Field Inflation
}

\author{
Karsten Jedamzik \\ Laboratoire de Physique Théorique et Astroparticules, UMR 5207-CNRS, \\ Université de Montpellier II, F-34095 Montpellier, France

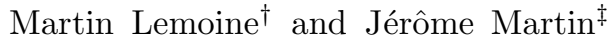 \\ Institut d'Astrophysique de Paris, \\ UMR 7095-CNRS, Université Pierre et Marie Curie, \\ 98bis boulevard Arago, 75014 Paris, France
}

(Dated: October 23, 2018)

\begin{abstract}
After cosmic inflation and before the transition to radiation domination, the cosmic energy density may have been dominated during an extended period by an oscillating massive scalar condensate. We show that during this period, sub-Hubble scale perturbations are subject to a metric preheating instability in the narrow resonance regime. We analyze in detail both, quadratic and quartic potentials. The instability leads to the growth of density perturbations which in many cases become non-linear already before the beginning of a radiation dominated Universe. This is particularly the case when requiring a phenomenologically preferred low reheat temperature. These early structures may lead to the emission of gravitational waves and the production of primordial black holes. Furthermore, it is not clear if they could modify the prediction of linear curvature perturbations on very large scales.
\end{abstract}

\section{INTRODUCTION}

The Universe having passed through an early inflationary epoch is by now a widely accepted paradigm for an explanation of apparent cosmic large-scale homogeneity as well as the perturbations observed in the cosmic microwave background radiation (CMBR). One of the earliest incarnations of inflation was given in Ref. [1] where a massive scalar field with potential $V(\phi)=m^{2} \phi^{2} / 2$, starting from super-Planck values, slowly rolls down towards its minimum thereby driving a period of accelerated expansion. Though many alternate scenarios of inflation have been discussed over the years, the above scenario of "chaotic inflation" remains one of the most successful in terms of reproducing the observed perturbations [2]. In particular, the predicted spectral index $n_{\mathrm{S}} \simeq 0.96$ in this model is consistent with that inferred from the WMAP7 analysis of the CMBR inhomogeneities, which gives $n_{\mathrm{S}} \simeq 0.963 \pm 0.012(68 \% \mathrm{CL})[\underline{3}]$.

After inflation has ended, cosmic energy density is dominated by an almost homogeneous oscillating inflaton field. A transition towards a radiation dominated Universe, as required to exist at the latest shortly before the epoch of Big Bang nucleosynthesis (BBN) which starts at cosmic temperature $T \sim 1 \mathrm{MeV}$, is usually assumed to occur by the perturbative decay of massive inflaton particles into radiation. This epoch is referred to as the epoch of reheating. Parametric resonance phenomena, inducing energy transfer to other scalars cou-

\footnotetext{
*Electronic address: jedamzik@lpta.univ-montp2.fr

${ }^{\dagger}$ Electronic address: lemoine@iap.fr

${ }^{\ddagger}$ Electronic address: jmartin@iap.fr
}

pled to the inflaton, referred to as preheating, though rich in their physics, have been shown to fail to provide a complete transition towards a radiation dominated Universe (see, e.g. [4, 5] for a review of preheating models). In case the inflaton would decay immediately after the end of chaotic inflation, and assuming $m^{2} \phi^{2} / 2$ inflation, the cosmic reheat temperature would be exceedingly large, i.e. $T_{\mathrm{rh}} \sim 10^{16} \mathrm{GeV}$. Such a large $T_{\mathrm{rh}}$ is phenomenologically problematic, as likely leading to the re-generation of unwanted relics, such as magnetic monopoles at a GUT transition, or massive gravitinos in supergravity [6, 7]. Modern constraints from BBN on unstable gravitinos constrain this reheating temperature down to $T_{\text {rh }} \lesssim 10^{7} \mathrm{GeV}$ [8 $[10]$. It is thus possible (and desirable) that between the end of chaotic inflation and reheating cosmic energy density is dominated for a very long expansion period by an almost homogeneous inflaton condensate. For example, for $T_{\text {rh }}$ as low as $\sim 1 \mathrm{MeV}$ the Universe would have expanded an immense factor $\sim 10^{25}$ between the end of inflation and reheating. It is such a cosmic era which is the topic of the current paper.

An inflaton oscillatory phase after inflation has received some attention with respect to the evolution of super-Hubble size perturbations [11 13]. It has been shown that, though one finds parametric resonance in the $m^{2} \phi^{2} / 2$ case, super-Hubble size perturbations thought to be responsible for the anisotropies in the CMBR stay constant in amplitude, as is the case in a radiation dominated Universe. It was therefore concluded that in single-field models "metric preheating" does not lead to non-trivial changes in the evolution of perturbations. However, as we will see in this paper (see also Ref. [11]), parametric resonance occurs even for sub-Hubble size regions, i.e. for all modes with physical wave vectors $k_{\text {phys }}<\sqrt{3 H m}$ (for a quadratic potential), where $H$ is the Hubble constant. 
In fact, these modes lie in the narrow resonance regime and the red-shifting is such that any mode which had once been resonant will always remain resonant. Due to this resonance the curvature perturbations of sub-Hubble modes stay constant rather than decreasing. One can readily show that this implies that the density contrast grows with scale factor $\delta \rho_{\boldsymbol{k}} / \rho \propto a$ implying non-linear cosmic structure formation after an expansion factor of $\sim 10^{6}$. This fact has so far received little attention in the literature.

Whether the Universe indeed entered an era with nonlinear structures, already before the epoch of reheating, depends on the reheat temperature and the scale of inflation. Attempting to keep the reheat temperature low enough to avoid overproduction of gravitinos, i.e. $T_{\mathrm{rh}} \approx 3 \times 10^{7}-3 \times 10^{8} \mathrm{GeV}[9$, 10] implies that essentially all large-field inflationary models are followed by a phase of non-linear structure formation. Figure 1 provides an illustration of this fact assuming a quadratic potential during the oscillating phase. It is seen that even for $H_{\text {end }}$ as low as $10^{7} \mathrm{GeV}$ non-linearities typically develop unless $T_{\mathrm{rh}} \gtrsim 10^{8} \mathrm{GeV}$. Therefore, a nonlinear cosmic phase before reheating appears to be a generic prediction. Note, however, that these considerations assume that after inflation energy density is dominated by a single oscillating scalar field.

These findings may not be surprising since it is known that the effective equation of state during such a phase is that of a Universe filled with pressure-less dust. One would thus expect the inflaton condensate to be gravitationally unstable, which indeed, has been shown in the Newtonian limit [14, 15] and in an averaged relativistic limit [16, 17]. However, our analysis and the effect that we report go beyond this Newtonian analogue, because this instability is found to be intrinsically some form of metric preheating. And, indeed, we find that a similar effect also takes place in the case of a quartic inflaton potential, for which the effective equation of state during oscillations is that of radiation. In this latter case, the resonance is (and remains) sharply peaked around the physical scale associated to the Hubble scale at the end of inflation, but the growth of the density contrast is exponential.

The outline of the paper is as follows. In Section II the metric preheating of scalar perturbations is analyzed in detail. The primordial power spectrum is calculated numerically over a wide range of scales. In Section III] we examine in detail the growth of density perturbations in a quadratic inflaton potential, while in Section IV we discuss the case of a quartic inflaton potential. Conclusions are drawn in Section $\nabla$

\section{GENERAL CONSIDERATIONS}

The evolution of scalar (density) perturbations is controlled by the so-called Mukhanov variable. If matter is described by a scalar field (as it is the case during in-

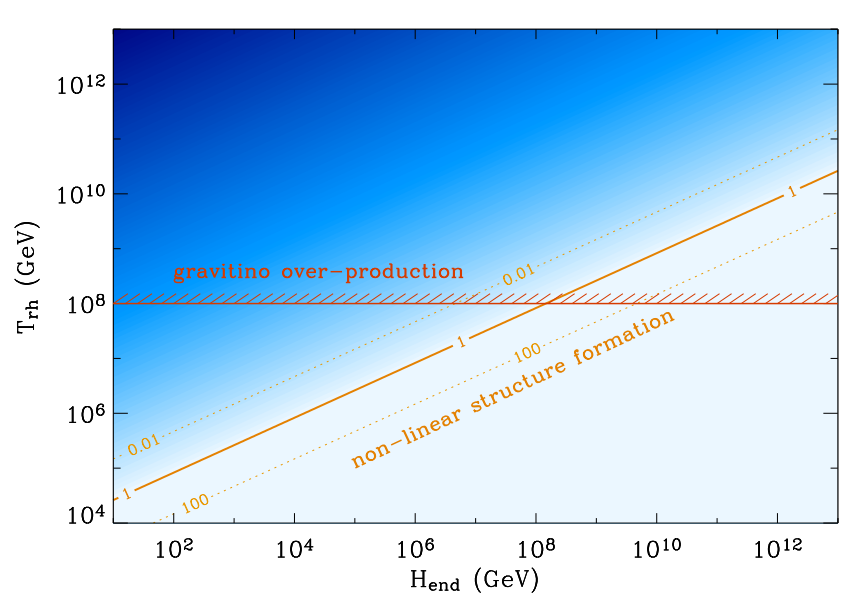

FIG. 1: Contour plot of the r.m.s. density fluctuation $\delta \rho_{\boldsymbol{k}} / \rho$ on the scale corresponding to the horizon at the end of inflation as measured at the onset of the radiation era in the $T_{\mathrm{rh}}$ (reheating temperature) $-H_{\text {end }}$ (Hubble constant at the end of inflation). The solid line that is labelled "1" delineates parameter space where non-linear structures form between the end of inflation and reheating (the region below this line) from parameter space where the Universe remains in the linear regime of density perturbations, i.e. $\delta \rho_{\boldsymbol{k}} / \rho \lesssim 1$, before reheating (the blue region above the line). The normalization for the curvature perturbations on the Hubble scale at the end of inflation has been assumed to be $\mathcal{P}_{\zeta} \sim 10^{-11}$ in accordance with Fig. 2 The two dotted lines indicate where the power spectrum of the fluctuations is larger and smaller than one or, equivalently, how the solid line would move when the normalization of the curvature perturbations on the Hubble scale after inflation is higher, $\mathcal{P}_{\zeta}=10^{-9}$ (line labeled 0.01), or lower, $\mathcal{P}_{\zeta}=10^{-13}$ (line labeled 100). The solid red line shows approximate upper limits on $T_{\mathrm{rh}}$ in order to avoid gravitino overproduction. Note that the figure is based on Eq. (25), and makes the fairly generic assumptions that energy density after inflation is dominated by a single oscillating scalar field with potential $m^{2} \phi^{2} / 2$, and that $\phi \lesssim M_{\mathrm{Pl}}$ at the end of inflation.

flation and preheating), then the equation of motion of the Fourier transform of Mukhanov's variable is given by [18 21]

$$
v_{\boldsymbol{k}}^{\prime \prime}+\left[k^{2}-\frac{\left(a \sqrt{\epsilon_{1}}\right)^{\prime \prime}}{a \sqrt{\epsilon_{1}}}\right] v_{\boldsymbol{k}}=0 .
$$

In this article a prime denotes a derivative with respect to conformal time while a dot means derivative with respect to cosmic time. The quantity $k$ is the comoving wave number and $\epsilon_{1} \equiv-\dot{H} / H^{2}$ is the first slow-roll parameter. The quantity $H=\dot{a} / a$ is the Hubble parameter and $a$ denotes the Friedman-Lemaitre-Robertson-Walker scale factor. Mukhanov's variable is related to the curvature perturbation,

$$
\zeta_{\boldsymbol{k}}=\sqrt{\frac{\kappa}{2}} \frac{v_{\boldsymbol{k}}}{a \sqrt{\epsilon_{1}}}
$$


where $\kappa \equiv 8 \pi / m_{\mathrm{Pl}}^{2}, m_{\mathrm{Pl}}$ being the (non-reduced) Planck mass. The importance of the curvature perturbation lies in the fact that, on large scales, it is a conserved quantity [22] and, therefore, it can be used to propagate the inflationary spectrum from the end of inflation to the post-inflationary era. As a consequence of the above relation, the spectrum of $\zeta_{\boldsymbol{k}}$ can be expressed as

$$
\mathcal{P}_{\zeta}(k) \equiv \frac{k^{3}}{2 \pi^{2}}\left|\zeta_{\boldsymbol{k}}\right|^{2}=\frac{2 k^{3}}{\pi m_{\mathrm{Pl}}^{2}}\left|\frac{v_{\boldsymbol{k}}}{a \sqrt{\epsilon_{1}}}\right|^{2} .
$$

In order to calculate $\mathcal{P}_{\zeta}(k)$, one needs to integrate Eq. (1), which requires the knowledge of the initial conditions for the mode function $v_{\boldsymbol{k}}$. Since, at the beginning of inflation, all the modes of astrophysical interest today were much smaller than the Hubble radius, the initial condition are chosen to be the Bunch-Davis vacuum which amounts to

$$
\lim _{k /(a H) \rightarrow+\infty} v_{k}=\frac{1}{\sqrt{2 k}} \mathrm{e}^{-i k \eta},
$$

where $\eta$ denotes conformal time.

The above formulation is well-adapted to the calculation of the power spectrum during inflation. However, in order to study the metric perturbations during the phase where the inflaton field oscillates it is more convenient to work in terms of cosmic time and to use the rescaled variable defined by $\tilde{v}_{\boldsymbol{k}}=a^{1 / 2} v_{\boldsymbol{k}}$. Then, Eq. (1) takes the form [13]

$$
\begin{aligned}
\ddot{\tilde{v}}_{\boldsymbol{k}} & +\left[\frac{k^{2}}{a^{2}}+\frac{\mathrm{d}^{2} V}{\mathrm{~d} \phi^{2}}+3 \kappa \dot{\phi}^{2}-\frac{\kappa^{2}}{2 H^{2}} \dot{\phi}^{4}+\frac{3 \kappa}{4}\left(\frac{\dot{\phi}^{2}}{2}-V\right)\right. \\
& \left.+2 \kappa \frac{\dot{\phi}}{H} \frac{\mathrm{d} V}{\mathrm{~d} \phi}\right] \tilde{v}_{\boldsymbol{k}}=0 .
\end{aligned}
$$

This formulation is particularly convenient as all the terms in the above equation remain non-singular even when the field oscillates at the bottom of its potential, that is to say when $\phi, \dot{\phi}$ and $\epsilon_{1}$ periodically vanish [12, 23].

To proceed further, one needs to choose the potential $V(\phi)$. The form of the inflaton potential is presently unknown (although it is constrained, see Refs. 24 26]). A convenient way to treat the most general case is to consider the following potential

$$
V(\phi)=V_{0} \pm \frac{1}{2} m^{2} \phi^{2}+\frac{\lambda}{4} \phi^{4}+\cdots,
$$

where the dots refer to higher order terms. As it is wellknown, this expansion can be difficult to control, most notably so for large field models where, during inflation, the vacuum expectation value of the field is large compared to the Planck mass. However, one can always assume that some terms in the above expansion are absent, for instance due to particular symmetries of the underlying theory. Different choices of coefficients in the above equation (6) correspond to different models. With the three terms displayed in Eq. (6), one can describe large field models, small field models and hybrid inflation (at least in the inflationary valley). In the next section, we consider the massive case.

\section{THE QUADRATIC CASE}

\section{A. Density Perturbations}

In this section, we investigate a model with a simple quadratic potential,

$$
V(\phi)=\frac{1}{2} m^{2} \phi^{2}
$$

We have in mind a large field inflationary model, but as discussed above, this quadratic potential has a more general applicability when it comes to discussing the physics of inflaton oscillations after the actual era of inflation. It may also describe the physics of curvaton oscillations. We will therefore keep wherever needed, the dependence of our results on the mass $m$ (and Hubble scale $H_{\text {end }}$ at the end of inflation), although we will use as fiducial values those corresponding to a large field inflationary model.

In large field models, the mass $m$ is fixed by the normalization of the amplitude of density fluctuations to that measured by COBE and WMAP. Standard calculations lead to

$$
m \simeq \frac{\sqrt{180 \pi}}{2 N_{*}+1} \frac{Q_{\mathrm{rms}-\mathrm{PS}}}{T} m_{\mathrm{Pl}}
$$

where $N_{*}$ is the number of e-folds before the end of inflation at which the scale of astrophysical interest left the Hubble radius during inflation (we take $N_{*}=50$ ) and where $Q_{\mathrm{rms}-\mathrm{PS}} / T \simeq 6 \times 10^{-6}$. This gives $m \simeq$ $1.4 \times 10^{-6} m_{\mathrm{Pl}}$.

The power spectrum $\mathcal{P}_{\zeta}(k)$ of density fluctuations at the end of inflation is calculated numerically in the following, via the integration of Eq. (1) throughout the inflationary era. Although one may find excellent approximations to this power spectrum on CMBR scales through the use of standard slow-roll calculations, we indeed find that up to second order in the slow-roll parameters, these methods cannot predict reliably the power spectrum on the shorter scales that we are interested in. More specifically, one finds to first order in the slow-roll parameter [27] 


$$
\mathcal{P}_{\zeta}(k)=\frac{H^{2}}{\pi \epsilon_{1} m_{\mathrm{Pl}}^{2}}\left[1-2(C+1) \epsilon_{1}-C \epsilon_{2}+\left(-2 \epsilon_{1}-\epsilon_{2}\right) \ln \left(\frac{k}{k_{*}}\right)\right],
$$

while, at second order, it is given by [27]

$$
\begin{aligned}
\mathcal{P}_{\zeta}(k)= & \frac{H^{2}}{\pi \epsilon_{1} m_{\mathrm{Pl}}^{2}}\left\{1-2(C+1) \epsilon_{1}-C \epsilon_{2}+\left(2 C^{2}+2 C+\frac{\pi^{2}}{2}-5\right) \epsilon_{1}^{2}+\left(C^{2}-C+\frac{7 \pi^{2}}{12}-7\right) \epsilon_{1} \epsilon_{2}\right. \\
& +\left(\frac{C^{2}}{2}+\frac{\pi^{2}}{8}-1\right) \epsilon_{2}^{2}+\left(-\frac{C^{2}}{2}+\frac{\pi^{2}}{24}\right) \epsilon_{2} \epsilon_{3}+\left[-2 \epsilon_{1}-\epsilon_{2}+2(2 C+1) \epsilon_{1}^{2}+(2 C-1) \epsilon_{1} \epsilon_{2}+C \epsilon_{2}^{2}-C \epsilon_{2} \epsilon_{3}\right] \\
& \left.\times \ln \left(\frac{k}{k_{*}}\right)+\frac{1}{2}\left(4 \epsilon_{1}^{2}+2 \epsilon_{1} \epsilon_{2}+\epsilon_{2}^{2}-\epsilon_{2} \epsilon_{3}\right) \ln ^{2}\left(\frac{k}{k_{*}}\right)\right\}
\end{aligned}
$$

In these expressions, $\epsilon_{2}$ and $\epsilon_{3}$ are slow-roll parameters belonging to the Hubble flow hierarchy 27, 28] $\epsilon_{n+1} \equiv \mathrm{d} \ln \epsilon_{n} /(\mathrm{d} N)$ ( $N$ being the number of e-folds) and $C$ is a numerical constant $C \simeq-0.7296$. The quantity $k_{*}$ is the so-called pivot scale the location of which is arbitrary but is usually chosen in the middle of the range of scales probed by the CMBR. These approximations fail to reproduce the power spectrum on small scales because the lever arm is then too important for the accuracy of the slow-roll calculation. This is rather unusual, as the slow-roll method generally provides an excellent approximation. This break-down of the slow-roll calculation on small scales is clearly seen in Fig. 2] which compares the full numerical calculation of the power spectrum with the above approximations for a large field model quadratic inflationary model. As one might expect, second-order slow-roll calculations are more accurate on small scales than the first order (which, among others justifies the techniques that have been developed for this purpose [29, 30]).

\section{Evolution of Density Perturbations after Inflation}

We are now interested in the behavior of perturbations after the end of inflation. On time scales $t \gg H_{\text {end }}^{-1}$ (the subscript "end" denotes hereafter quantities evaluated at the end of inflation), the inflaton vacuum expectation value evolves approximately as

$$
\phi(t) \simeq \phi_{\mathrm{end}}\left(\frac{a_{\mathrm{end}}}{a}\right)^{3 / 2} \sin (m t)
$$

for a quadratic inflaton potential. Let us recall that $\phi_{\text {end }} \simeq m_{\mathrm{Pl}} /(2 \sqrt{\pi})$ and $H_{\text {end }} \simeq m / \sqrt{3}$ in the particular case of a large field quadratic inflationary model. Plugging Eq. (11) into Eq. (5), it is easy to see that the dominant term of the last four gravitational terms is the last one as it scales as $1 / a^{3 / 2}$ while the others decay as $1 / a^{3}$. Neglecting the other three terms one finds an equation

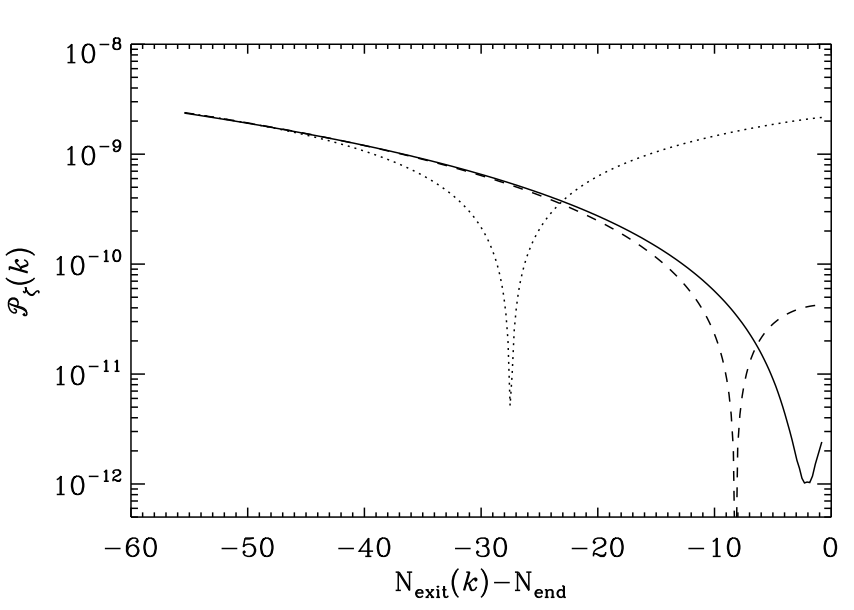

FIG. 2: Primordial power spectrum of curvature perturbations (solid line) for the large field model $V(\phi)=m^{2} \phi^{2} / 2$. Here $N_{\text {exit }}(k)-N_{\text {end }}$ is $\ln \left[a_{\text {cross }}(k) / a_{\text {end }}\right]$, with $a_{\text {cross }}(k)$ the scale factor at Hubble radius crossing of mode $k$ during inflation (i.e. $\left.k / a_{\text {cross }} \equiv 2 \pi H\right)$, and $a_{\text {end }}$ the scale factor at the end of inflation. The dotted and dashed lines respectively represents the first and second order slow-roll results as given by Eqs. (9) and (10) with $\epsilon_{1} \simeq 1 /\left[2\left(N_{*}+1 / 2\right)\right]$, $\epsilon_{2}=\epsilon_{3}=1 /\left(N_{*}+1 / 2\right)$ and $N_{*} \simeq 50$. The slow-roll result is accurate in the CMBR region but breaks down on smaller scales where the predicted power spectra become negative. The upturn is related to modes that have not crossed out the Hubble radius and have remained in their vacuum state, see text for details.

of motion of the form

$$
\frac{\mathrm{d}^{2} \tilde{v}_{k}}{\mathrm{~d} z^{2}}+\left[1+\frac{k^{2}}{m^{2} a^{2}}-\sqrt{6 \kappa} \phi_{\text {end }}\left(\frac{a_{\text {end }}}{a}\right)^{3 / 2} \cos (2 z)\right] \tilde{v}_{\boldsymbol{k}}=0,
$$

where we have defined $z \equiv m t+\pi / 4$. This equation is similar to a Mathieu equation

$$
\frac{\mathrm{d}^{2} \tilde{v}_{\boldsymbol{k}}}{\mathrm{d} z^{2}}+\left[A_{\boldsymbol{k}}-2 q \cos (2 z)\right] \tilde{v}_{\boldsymbol{k}}=0
$$




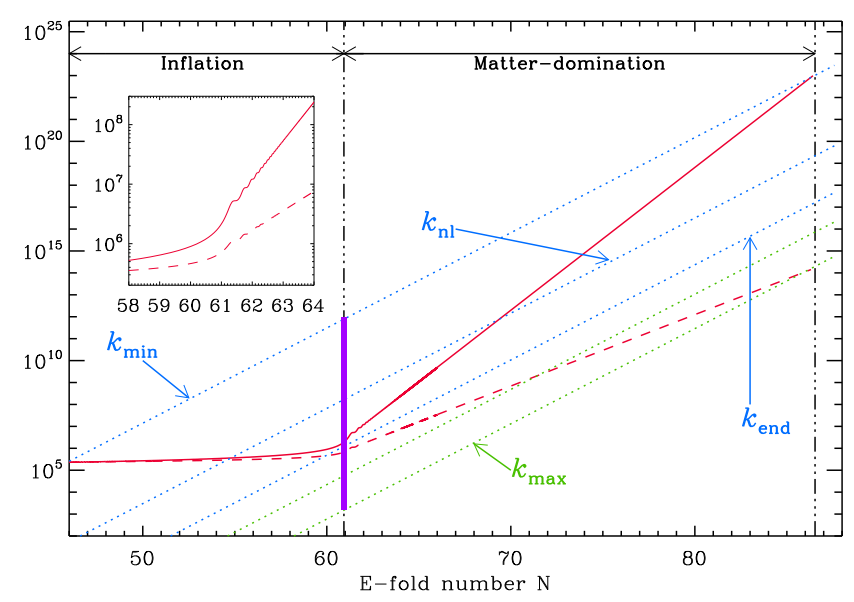

FIG. 3: Hubble radius $\ell_{\mathrm{H}}$ (red solid line), lower bound of resonance band $\ell_{\mathrm{C}}$ (red dashed line) and physical wavelengths (blue and green dotted lines), in Planck units, versus the efold number during the final stage of inflation and the inflaton oscillation era for a large field $m^{2} \phi^{2} / 2$ model. The reheating temperature is $T_{\mathrm{rh}}=10^{7} \mathrm{GeV}$ hence the number of e-folds during the inflaton oscillation era is $N_{\mathrm{rh}} \simeq 25.6$. The inset shows the detailed behaviors of $\ell_{\mathrm{H}}$ and $\ell_{\mathrm{C}}$ at the transition between inflation and inflaton oscillation.

with

$$
\begin{aligned}
A_{k} & =1+\frac{k^{2}}{m^{2} a^{2}} \\
q & =\frac{\sqrt{6 \kappa}}{2} \phi_{\text {end }}\left(\frac{a_{\text {end }}}{a}\right)^{3 / 2} .
\end{aligned}
$$

Since $q \ll 1$, we are in the narrow resonance regime. The first instability band is given by $1-q<A_{k}<1+q$ which amounts to

$$
0<\frac{k}{a}<\sqrt{3 H m} .
$$

From the above inequality, we note the emergence of a new characteristic spatial length given by $\ell_{\mathrm{C}} \equiv 1 / \sqrt{3 H m}$, which sets the lower bound to the range of resonant spatial scales. During inflation, in a large field model, $\ell_{\mathrm{C}}$ is almost a constant and $\ell_{\mathrm{C}} \sim \ell_{\mathrm{H}}$, with $\ell_{\mathrm{H}} \equiv H^{-1}$. After the end of inflation, during the inflaton oscillation era, with $H \propto a^{-3 / 2}$ one has $\ell_{\mathrm{C}} \propto a^{3 / 4}$ and, therefore, $\ell_{\mathrm{C}}$ grows more slowly than the Hubble radius $\ell_{\mathrm{H}} \propto a^{3 / 2}$. The situation is summarized in Fig. 3 where both scales are displayed (solid and dashed red lines). Their evolution has been computed by an exact numerical integration of the equations of motion in the case of a large field model. Since the physical wavelength of a comoving Fourier mode, $\lambda \propto a$, grows faster than $\ell_{\mathrm{C}}$, the scales may cross $\ell_{\mathrm{C}}$ during inflation or during the matter-dominated era and thereby enter the instability band. Furthermore, a mode that has entered this band will always remain in it, at least until reheating is completed. One can thus distinguish two types of modes: those that have entered the resonance band during inflation (blue lines in Fig. 3) and those that have entered it during the inflaton oscillation era (green lines in Fig. 31). Of course, the preheating instability is only operative after inflation and before reheating.

Having identified the scales affected by the resonance, we now discuss their amplification. For the first instability band, the Floquet index is given by $\mu=q / 2$. Then the mode function evolves as $\tilde{v}_{\boldsymbol{k}} \propto \mathrm{e}^{\mu z}$. However, in the present case, we have a time-dependent Floquet index, for which the corresponding solution can be written as 13.

$$
\tilde{v}_{\boldsymbol{k}} \propto \exp \left(\int \mu \mathrm{d} z\right) \propto a^{3 / 2} .
$$

Note that, taking the integral of the time-dependent $\mu$ does not correspond to the WKB approximation, but rather is an educated guess confirmed by numerical simulation, see below. This implies that $v_{\boldsymbol{k}} \propto a$ and therefore $\zeta_{\boldsymbol{k}}$ is constant according to its definition Eq. (2). Note also that the curvature perturbation usually remains constant only on super-Hubble scales while decaying on subHubble scales, unless there is gravitational instability.

We are now interested in the behavior of the fractional mass-energy density perturbation, $\delta_{\boldsymbol{k}}(\eta) \equiv \delta \rho_{\boldsymbol{k}} / \rho$, where $\rho$ is the background energy density of the scalar field. The perturbed Einstein equation implies that

$$
\delta_{\boldsymbol{k}}=-\frac{2}{3}\left(\frac{k^{2}}{a^{2} H^{2}}+3\right) \Phi_{\boldsymbol{k}}-2 \frac{\dot{\Phi}_{\boldsymbol{k}}}{H},
$$

where $\Phi_{\boldsymbol{k}}$ is the Bardeen potential [18, 31]. The Bardeen potential is related to the quantity $\zeta_{\boldsymbol{k}}$ by

$$
\zeta_{\boldsymbol{k}}=\frac{2}{3} \frac{H^{-1} \dot{\Phi}_{\boldsymbol{k}}+\Phi_{\boldsymbol{k}}}{1+p / \rho}+\Phi_{\boldsymbol{k}}
$$

where $p$ denotes the pressure. For constant $p / \rho$, if $\Phi_{\boldsymbol{k}}$ is constant so is $\zeta_{\boldsymbol{k}}$. This is a generic result on large scales in one fluid models. If $\Phi_{\boldsymbol{k}}$ evolves as a power-law in the scale factor (also a generic result), $\Phi_{k} \propto a^{s}$, then $H^{-1} \dot{\Phi}_{\boldsymbol{k}}=s \Phi_{\boldsymbol{k}}$ and consequently, Eq. (19) indicates that $\zeta_{\boldsymbol{k}}$ follows the same power-law behavior. Therefore, the constancy of $\zeta_{\boldsymbol{k}}$ in the resonance band implies that $\Phi_{\boldsymbol{k}}$ also remains constant. This is confirmed by a numerical integration of the equations of motion, see Fig. 4. Then $\zeta_{\boldsymbol{k}}=5 \Phi_{\boldsymbol{k}} / 3$, as the pressure vanishes on average during the preheating stage. Consequently, one may express $\delta_{\boldsymbol{k}}$ as a function of $\zeta_{k}$ as follows

$$
\delta_{\boldsymbol{k}}=-\frac{2}{5}\left(\frac{k^{2}}{a^{2} H^{2}}+3\right) \zeta_{\boldsymbol{k}} .
$$

This relation remains valid in both the large scale and the small scale limit (with respect to the Hubble radius) provided the modes are in the instability band (i.e. $\zeta_{\boldsymbol{k}}$ is constant). Given that $a H \propto a^{-1 / 2}$ in a inflaton oscillation dominated Universe, one then finds that $\delta_{\boldsymbol{k}}$ grows as 


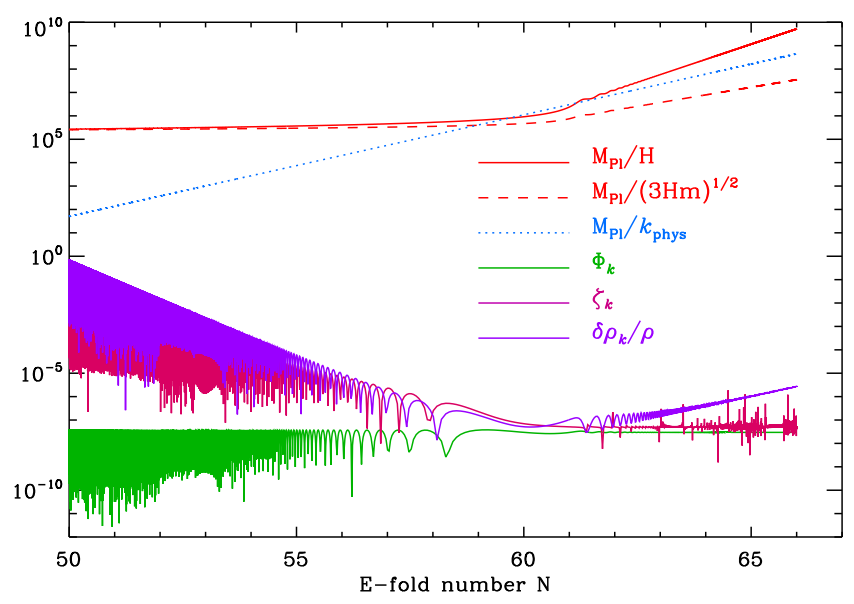

FIG. 4: Evolution of the density perturbations $\delta \rho_{\boldsymbol{k}} / \rho, \zeta_{\boldsymbol{k}}$ and $\Phi_{\boldsymbol{k}}$ towards the end of inflation and during the ensuing period of metric preheating after inflation. The notation is as in the text with $k_{\text {phys }} \equiv k / a ; N$ denotes the e-fold number of the scale factor. The reduced Planck mass $M_{\mathrm{Pl}} \equiv m_{\mathrm{Pl}} / \sqrt{8 \pi}$ is used in this plot.

$a(t)$ on sub-Hubble scales but remains constant on superHubble scales. This behavior is also confirmed by the numerical integration whose results are shown in Fig. 4. The preheating instability for all sub-Hubble modes with $k / a<\sqrt{3 H m}$ may thus be understood as the gravitational instability of the oscillating scalar condensate.

Let us summarize our main findings. We have found that all modes in the instability band and outside the Hubble radius are such that $\zeta_{\boldsymbol{k}}$ and $\delta_{\boldsymbol{k}}$ are constant. This is of course the case for modes on scales probed by the CMBR and, therefore, one recovers the fact that the inflationary power spectrum propagates through the reheating stage without being affected. However, for those modes which are in the instability band and inside the Hubble radius, one finds that $\zeta_{\boldsymbol{k}}$ is constant but that $\delta_{\boldsymbol{k}}$ grows as $a(t)$. Therefore, and contrary to the standard lore, the preheating stage can affect the behavior of the perturbations even if there is only one non-interacting fluid.

\section{Spectrum of Density Perturbations at Reheating}

From the above considerations, we see that the amplification described above occurs only for a range of scales, $k \in\left[k_{\min }, k_{\max }\right]$ in terms of comoving wavenumber. We now discuss this point in more detail and specify $k_{\min }$ and $k_{\max }$. Henceforth, we introduce the short-hand notation:

$$
\hat{k} \equiv \frac{k}{a_{\text {end }} H_{\text {end }}},
$$

where the label "end" refers as before to the end of inflation. The smallest unstable spatial scale is that which enters the resonance band at the time of completion of reheating. It corresponds to the green dotted line labelled " $k_{\max }$ " in Fig. 3. Interestingly enough, this scale has never been super-Hubble. From the condition $k_{\text {max }} / a_{\mathrm{rh}}=\sqrt{3 H_{\mathrm{rh}} m}$, where $H_{\mathrm{rh}}$ is the Hubble parameter at the end of the reheating epoch or at the beginning of the radiation dominated phase, one obtains

$$
\begin{aligned}
\hat{k}_{\max }= & 1.37 \times 10^{3}\left(\frac{m}{1.4 \times 10^{-6} m_{\mathrm{Pl}}}\right)^{1 / 2} \\
& \times\left(\frac{T_{\mathrm{rh}}}{10^{7} \mathrm{GeV}}\right)^{-1 / 3}\left(\frac{H_{\mathrm{end}}}{10^{13} \mathrm{GeV}}\right)^{-1 / 3} .
\end{aligned}
$$

The last equation assumes $g_{*}=230$ degrees of freedom at reheating. All modes with $\hat{k}<\hat{k}_{\max }$ have entered the resonance band during inflation or during the preheating era.

We have also seen that a necessary condition for the growth of $\delta_{\boldsymbol{k}}$ on a given scale is that the corresponding mode lies within the Hubble radius at some time before the reheating epoch. As a consequence, the largest spatial scale that is unstable with respect to the growth of $\delta_{\boldsymbol{k}}$ is that which re-enters the Hubble radius at the end of the pre-reheating epoch, i.e. $k_{\min } \simeq a_{\mathrm{rh}} H_{\mathrm{rh}}$. This corresponds to the blue dotted line labeled " $k_{\min }$ " in Fig. 3 and

$$
\hat{k}_{\text {min }}=2.74 \times 10^{-6}\left(\frac{T_{\mathrm{rh}}}{10^{7} \mathrm{GeV}}\right)^{2 / 3}\left(\frac{H_{\mathrm{end}}}{10^{13} \mathrm{GeV}}\right)^{-1 / 3}
$$

As the density contrast increases in direct proportion to the scale factor for modes in the range $k \in\left[k_{\min }, k_{\max }\right]$, non-linear structures may eventually form. The amplitude of the density contrast at the end of the preheating epoch is given by the density contrast at the end of inflation and by the e-fold number of growth while the mode is sub-horizon sized and in the resonance band. At this point, one should distinguish the modes that enter the resonance band during inflation (the dotted blue lines in Fig. 31) and those that have entered the band during the preheating era but that have never exited the Hubble radius (the dotted green lines in Fig. 3).

We first address the former ones, for which $k \in$ $\left[k_{\text {min }}, k_{\text {end }}\right]$, with $\hat{k}_{\text {end }} \equiv 1$. Given that, in the resonance band, the Fourier density contrast remains constant on super-Hubble scales, the behavior of $\delta_{\boldsymbol{k}}$ on sub-Hubble scale can be approximated as

$$
\delta_{\boldsymbol{k}}=\delta_{\boldsymbol{k}}^{\mathrm{hc}} \frac{a}{a_{\mathrm{hc}}}=\delta_{\boldsymbol{k}}^{\mathrm{end}} \frac{a}{a_{\mathrm{hc}}},
$$

where the label "hc" refers to the epoch of Hubble radius crossing of mode $\boldsymbol{k}$ and $\delta_{\boldsymbol{k}}^{\text {end }}$ is the density contrast calculated at the end of inflation, see Fig. 2, Let us precise the value of $a_{\mathrm{hc}}$ : in order to match both asymptotic behaviors of $\delta_{\boldsymbol{k}}$ in the super-horizon, namely $\delta_{\boldsymbol{k}} \rightarrow 6 \zeta_{\boldsymbol{k}} / 5$ and in the sub-horizon regimes, namely $\delta_{\boldsymbol{k}} \rightarrow[k /(a H)]^{2} 2 \zeta_{\boldsymbol{k}} / 5$, one defines $a_{\mathrm{hc}}$ as that at which $k=\sqrt{3} a_{\mathrm{hc}} H_{\mathrm{hc}}$. This 
implies that $a_{\mathrm{hc}}=3 a_{\mathrm{end}} \hat{k}^{-2}$. Now, using Eq. (20), one can write $\delta_{\boldsymbol{k}}^{\text {end }}=(6 / 5) \sqrt{2 \pi^{2} \mathcal{P}_{\zeta}(k) / k^{3}}$. Then, the power spectrum of $\delta_{k}$ at the time of reheating is evaluated as

$$
\begin{aligned}
\mathcal{P}_{\delta}^{\mathrm{rh}}(k)= & \frac{k^{3}}{2 \pi^{2}}\left|\delta_{\boldsymbol{k}}^{\mathrm{end}}\right|^{2}\left(\frac{a_{\mathrm{rh}}}{a_{\mathrm{hc}}}\right)^{2} \\
= & 2.82 \times 10^{10} \hat{k}^{4}\left(\frac{\mathcal{P}_{\zeta}}{10^{-11}}\right)\left(\frac{T_{\mathrm{rh}}}{10^{7} \mathrm{GeV}}\right)^{-8 / 3} \\
& \times\left(\frac{H_{\mathrm{end}}}{10^{13} \mathrm{GeV}}\right)^{4 / 3} .
\end{aligned}
$$

The variance of the density fluctuation enclosing the scale defined by $k$ is directly expressed at reheating as

$$
\left\langle\delta^{2}\right\rangle_{k}=\int_{0}^{k} \mathrm{~d} \ln k^{\prime} \mathcal{P}_{\delta}^{\mathrm{rh}}\left(k^{\prime}\right),
$$

and the condition $\left\langle\delta^{2}\right\rangle_{k} \simeq 1$ thus roughly amounts to $\mathcal{P}_{\delta}^{\text {rh }} \simeq 1$. This singles out a specific spatial scale (corresponding to wavenumber $k_{\mathrm{nl}}$ ), such that scales with $k<k_{\text {nl }}$ have not had sufficient time to become nonlinear, while those with $k>k_{\mathrm{nl}}$ have become non-linear by reheating. Straightforward manipulations lead to

$$
\begin{aligned}
\hat{k}_{\mathrm{nl}} \simeq & 4.50 \times 10^{-3}\left(\frac{\mathcal{P}_{\zeta}}{10^{-11}}\right)^{-1 / 4}\left(\frac{T_{\mathrm{rh}}}{10^{7} \mathrm{GeV}}\right)^{2 / 3} \\
& \times\left(\frac{H_{\text {end }}}{10^{13} \mathrm{GeV}}\right)^{-1 / 3} .
\end{aligned}
$$

Let us now address the unstable modes that have always been sub-Hubble, namely $k \in\left[k_{\text {end }}, k_{\text {max }}\right]$. For these modes, Mukhanov's variable is still given by the vacuum solution, Eq. (4), until it enters the instability band. This leads to

$$
\zeta_{\boldsymbol{k}}=\frac{\mathrm{e}^{-i k\left(\eta-\eta_{\mathrm{end}}\right)}}{\sqrt{\epsilon_{1}}}\left(\frac{a_{\mathrm{end}}}{a}\right) \zeta_{\boldsymbol{k}}^{\mathrm{end}},
$$

where $\epsilon_{1} \sim 3 / 2$ in the preheating era. In order to derive this expression, we have set $\epsilon_{1}=1$ at the end of inflation and we have assumed that Mukhanov's variable has remained constant through the (instantaneous) transition from inflation to the preheating era; this is expected insofar as the modes are well sub-Hubble at that time, $k \gg a H$, hence Mukhanov's variable oscillates freely. The quantity $\zeta_{\boldsymbol{k}}^{\text {end }}$ represents the value of $\zeta_{\boldsymbol{k}}$ at the end of inflation or at the onset of the oscillatory phase. Using Eq. (19) with $p=0$, one finds $\zeta_{\boldsymbol{k}}=\Phi_{\boldsymbol{k}}$ since $\zeta_{\boldsymbol{k}}$ and $\Phi_{k}$ must share the same power-law behavior $\propto a^{-1}$ (as discussed above), hence $H^{-1} \dot{\Phi}_{k}=-\Phi_{k}$. Then, Eq. (18) leads to

$$
\begin{aligned}
\delta_{\boldsymbol{k}} & =-\frac{2}{3} \frac{k^{2}}{a^{2} H^{2}} \Phi_{\boldsymbol{k}} \\
& =-\frac{2 \hat{k}^{2}}{3} \frac{\mathrm{e}^{-i k\left(\eta-\eta_{\text {end }}\right)}}{\sqrt{\epsilon_{1}}} \zeta_{\boldsymbol{k}}^{\text {end }} \\
& =C(k) \mathrm{e}^{-i k \eta} .
\end{aligned}
$$

The density contrast then oscillates with a constant amplitude until the mode enters the resonance band, in which $\delta_{\boldsymbol{k}}=C(k) a / a_{\mathrm{rc}}$ where $a_{\mathrm{rc}}$ denotes the scale factor at the entry into the resonance band. This latter is given by $a_{\mathrm{rc}}=k^{4} /\left(9 m^{2} a_{\text {end }}^{3} H_{\text {end }}^{2}\right)$ which implies that $a_{\mathrm{rh}} / a_{\mathrm{rc}}=\left(\hat{k}_{\max } / \hat{k}\right)^{4}$. The power spectrum of density fluctuations at the time of preheating can now be computed as

$$
\mathcal{P}_{\delta}^{\mathrm{rh}}(k)=\frac{8 \hat{k}^{4}}{27}\left(\frac{\hat{k}_{\mathrm{max}}}{\hat{k}}\right)^{8} \mathcal{P}_{\zeta}^{\mathrm{end}}(k) .
$$

The quantity $\mathcal{P}_{\zeta}^{\text {end }}$ is the power spectrum at the end of inflation for the modes that have never exited the Hubble radius,

$$
\mathcal{P}_{\zeta}^{\text {end }}(k)=\frac{\hat{k}^{2}}{\pi}\left(\frac{H_{\text {end }}}{m_{\mathrm{Pl}}}\right)^{2} .
$$

We can check that it gives the correct order of magnitude by comparing with the exact result in Fig. 2 for the case of a large field model. Inserting this expression in Eq. (30), we see that the spectrum now decreases as $\hat{k}^{-2}$ while it was proportional to $\hat{k}^{4}$ in the other range of wavenumbers $\left[k_{\min }, k_{\text {end }}\right]$ that we discussed previously.

Finally, for those modes that have never entered the resonance band and never crossed out the Hubble radius, that is to say $\hat{k}>\hat{k}_{\max }$, the density contrast always behaves according to Eq. (29) and, therefore, the corresponding power spectrum is given by

$$
\mathcal{P}_{\delta}^{\mathrm{rh}}(k)=\frac{8 \hat{k}^{4}}{27} \mathcal{P}_{\zeta}(k) .
$$

This spectrum is proportional to $k^{6}$. Of course, these modes are still in a vacuum state and therefore, despite the fact that the vacuum fluctuations are large (which is likely to be linked to the pathological behavior of the theory in the ultra-violet regime), there is no energy density associated to this phenomenon. One way to see this is to compute the evolution of the modes, and in particular the Bogoliubov coefficient $\beta_{\boldsymbol{k}}$ of the negative frequency WKB branch at late times, on sub-horizon scales (assuming the vacuum corresponds to the positive frequency branch). For these vacuum modes, $\beta_{\boldsymbol{k}}=0$ because the WKB approximation has always remained valid. The energy density stored in these modes, which is given by

$$
\frac{\mathrm{d} \delta \rho_{\boldsymbol{k}}}{\mathrm{d} \ln \hat{k}} \simeq \frac{k^{4}}{2 \pi^{2} a^{4}}\left|\beta_{\boldsymbol{k}}\right|^{2},
$$

consequently vanish.

The situation is summarized in Fig. 2 where the spectrum $\mathcal{P}_{\delta}(k)$ is displayed in the different regimes studied before. The striking feature of this plot is of course that, for $k>k_{\mathrm{nl}}$, the power spectrum is non-linear at the time of reheating; let us stress that of course, the plotted values only represent the extrapolation of the linear calculation. 


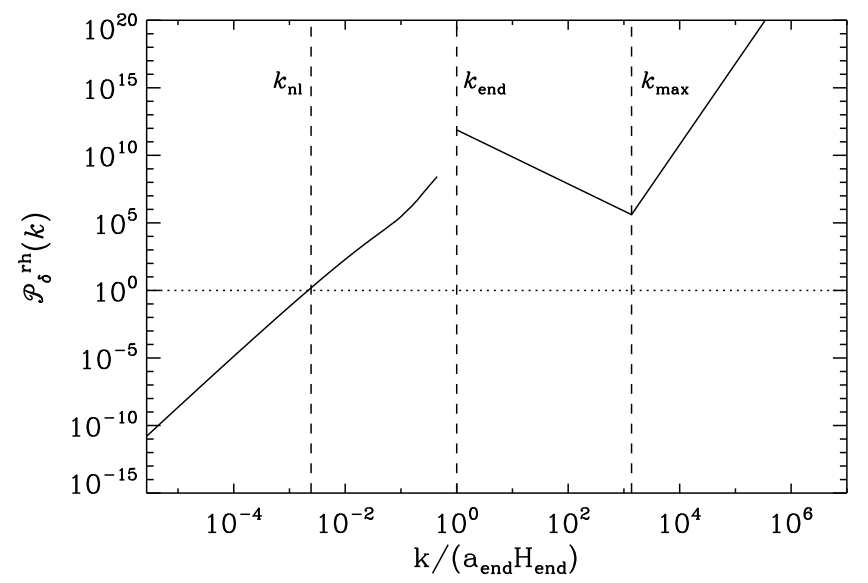

FIG. 5: Power Spectrum $\mathcal{P}_{\delta}^{\mathrm{rh}}(k)$, computed at reheating as given by Eqs. (25), (29) and (32). In the range $k \in\left[k_{\text {min }}, k_{\text {end }}\right]$, the spectrum is evaluated numerically, see Fig. 2 . The small discontinuity observed at $k_{\text {end }}$ is just a numerical artefact, the actual power spectrum being of course continuous.

The non-linearity of modes with $k>k_{\text {end }}$ is rather intriguing, as these modes have never crossed out the Hubble radius, hence one might question their classical nature. However, it is rather straightforward to show that for these modes, $\left|\beta_{\boldsymbol{k}}\right| \sim 1$, hence energy density is indeed stored on those scales. From this point of view, the non-linearities observed in the power spectrum should a priori be taken seriously and viewed as a sign that structure formation has started on those scales. In order to assess the nature of these modes, one should apply the discussion of the so-called quantum to classical transition of modes [21, 32] to the present case. However, this question is beyond the scope of this paper.

\section{Associated Mass Scales}

Finally, it is interesting to quantify the range of masses associated with the collapsing structures. In terms of the wavenumber of the perturbation, the enclosed mass can be written as

$$
M=\frac{4 \pi}{3}(2 \pi)^{3} \frac{\rho_{\mathrm{end}}}{H_{\mathrm{end}}^{3}} \hat{k}^{-3}
$$

According to the previous considerations, the mass corresponding to maximum growth of the density fluctuations corresponds to the scale $k_{\text {end }}$. Using the above expression, straightforward manipulations lead to the following expression

$$
M_{\mathrm{end}} \simeq 3.3 \times 10^{3}\left(\frac{H_{\mathrm{end}}}{10^{13} \mathrm{GeV}}\right)^{-1} \mathrm{~g} .
$$

The minimal mass, associated to $k_{\max }$ is given by

$$
\begin{aligned}
M\left(k_{\max }\right) \simeq & 1.3 \times 10^{-6}\left(\frac{T_{\mathrm{rh}}}{10^{7} \mathrm{GeV}}\right) \\
& \times\left(\frac{m}{1.4 \times 10^{-6} m_{\mathrm{Pl}}}\right)^{-3 / 2} \mathrm{~g} .
\end{aligned}
$$

The maximum mass is associated to the scale $k_{\mathrm{nl}}$, i.e.

$$
M_{\mathrm{nl}} \simeq 3.6 \times 10^{10}\left(\frac{\mathcal{P}_{\zeta}}{10^{-11}}\right)^{3 / 4}\left(\frac{T_{\mathrm{rh}}}{10^{7} \mathrm{GeV}}\right)^{-2} \mathrm{~g} .
$$

Quite interestingly, the abundance of primordial black holes with mass $\lesssim 10^{9} \mathrm{~g}$ is constrained by early Universe cosmology. Such black holes indeed evaporate before BBN has started and the black hole temperature is high enough to allow the production of copious amounts of lightest supersymmetric particles, in excess of the closure density [33], as well as gravitinos and other late time decaying massive particles, whose decay products would ruin the success of BBN 34, 35]. If such black holes leave Planck-mass relics at their final point of evaporation, additional constraints can be obtained from closure density arguments [36]. For black hole masses $\gtrsim 10^{9} \mathrm{~g}$, stringent constraints can be derived from the impact of the evaporated radiation on $\mathrm{BBN}$, see [37] for a detailed discussion of this and other observational constraints.

\section{B. Tensor modes}

In the previous section, we have studied the amplification of density perturbations during the reheating epoch. Naturally, one may wonder whether tensor modes can be amplified through a similar instability. Their Fourier amplitude $h_{\boldsymbol{k}}=\mu_{\boldsymbol{k}} / a$ obeys the equation [38]

$$
\mu_{\boldsymbol{k}}^{\prime \prime}+\left(k^{2}-\frac{a^{\prime \prime}}{a}\right) \mu_{\boldsymbol{k}}=0 \text {. }
$$

The effective potential is thus much simpler than in the case of density perturbations as it only involves the scale factor and its time derivatives up to second order. Defining $\bar{\mu}_{\boldsymbol{k}} \equiv a^{1 / 2} \mu_{\boldsymbol{k}}$ and working in terms of the cosmic time, one obtains

$$
\ddot{\bar{\mu}}_{\boldsymbol{k}}+\left(\frac{k^{2}}{a^{2}}-\frac{3 \dot{H}}{2}-\frac{9}{4} H^{2}\right) \bar{\mu}_{\boldsymbol{k}}=0 .
$$

Using the explicit form of the inflaton field given by Eq. (11), the equation of motion takes the form

$$
\frac{\mathrm{d}^{2} \bar{\mu}_{\boldsymbol{k}}}{\mathrm{d} z^{2}}+\left[\frac{k^{2}}{a^{2} m^{2}}+\frac{3 \kappa}{8} \phi_{\text {end }}^{2}\left(\frac{a_{\text {end }}}{a}\right)^{3} \cos (2 z)\right] \bar{\mu}_{\boldsymbol{k}}=0,
$$

where $z \equiv m t$. Therefore, one also obtains a Mathieu equation with

$$
\begin{aligned}
A_{\boldsymbol{k}} & =\frac{k^{2}}{a^{2} m^{2}} \\
q & =-\frac{3 \kappa}{16} \phi_{\mathrm{end}}^{2}\left(\frac{a_{\mathrm{end}}}{a}\right)^{3} .
\end{aligned}
$$


These equations should be compared to Eqs. (14) and (15). We are still in the regime $q \ll 1$ but the crucial point is that there is no term 1 in the expression of $A_{\boldsymbol{k}}$ in Eq. (41). This means that, for a given scale, the condition $1-q<A_{\boldsymbol{k}}<1+q$ cannot be maintained since $A_{k}$ does not scale as $q$ does. Therefore the amplification does not occur for gravitational waves.

\section{THE QUARTIC CASE}

\section{A. A quartic potential $V(\phi)=\lambda \phi^{4} / 4$}

In the previous section, we have studied a large field model with a quadratic potential. This corresponds to a pretty generic situation since, close to its minimum, the inflaton potential can always be approximated by a parabola. However, it might also be that, for some unspecified reason (generically, the presence of a particular symmetry), the quadratic term in the Taylor expansion (6) is absent. Then, one has to repeat the previous analysis in the case where the potential is given by

$$
V(\phi)=\frac{\lambda}{4} \phi^{4} .
$$

In the following, we find that growth also occurs for this case. This result is particularly interesting, as it shows that the growth of density perturbations goes beyond the simple analogue of Newtonian collapse in a dust Universe. Indeed, in the case of $\lambda \phi^{4} / 4$, the effective equation of state is that of radiation.

As before, we assume that the potential is approximated by the above quartic form in the preheating era without making any specific assumption on the actual potential during the inflationary era. We note that quartic inflation can be normalized to COBE anisotropies with $\lambda \simeq 10^{-12}$, but that at the same time such an inflationary potential is disfavored by detailed analysis of CMB data [24].

For a quartic potential, the evolution of the inflaton field around its minimum can be expressed in the large time limit $t \gg H_{\text {end }}^{-1}$ as [4, 5, 39, 40]

$$
\phi(\eta)=\phi_{\text {end }}\left(\frac{a_{\text {end }}}{a}\right) \operatorname{cn}\left(x-x_{\text {end }} ; \frac{1}{\sqrt{2}}\right),
$$

where $x \equiv a_{\text {end }} \phi_{\text {end }} \sqrt{\lambda} \eta, \eta$ being the conformal time and cn denotes the Jacobi cosine function [41, 42]. This solution should be inserted in Eq. (5D). Then, keeping only the dominant terms, the equation that controls the evolution of Mukhanov's variable reduces to

$$
\begin{aligned}
\ddot{\tilde{v}}_{\boldsymbol{k}}+ & {\left[\frac{k^{2}}{a^{2}}+3 \lambda \phi_{\text {end }}^{2}\left(\frac{a_{\text {end }}}{a}\right)^{2}\right.} \\
& \left.\times \operatorname{cn}^{2}\left(x-x_{\text {end }} ; \frac{1}{\sqrt{2}}\right)\right] \tilde{v}_{\boldsymbol{k}}=0 .
\end{aligned}
$$

It is more convenient to change back to the variable $v_{\boldsymbol{k}}$ and to express this equation in terms of the variable $x$ defined above:

$$
\frac{\mathrm{d}^{2} v_{\boldsymbol{k}}}{\mathrm{d} x^{2}}+\left[\frac{k^{2}}{\phi_{\text {end }}^{2} a_{\text {end }}^{2} \lambda}+3 \mathrm{cn}^{2}\left(x-x_{\text {end }} ; \frac{1}{\sqrt{2}}\right)\right] v_{\boldsymbol{k}}=0 .
$$

As emphasized in Refs. [5, 39, 40], this equation is the so-called Lamé equation for which the Floquet chart is exactly known. There is amplification if $3 / 2<$ $k^{2} /\left(\phi_{\text {end }}^{2} a_{\text {end }}^{2} \lambda\right)<\sqrt{3}$. Using $H_{\text {end }}^{2} \simeq \kappa \lambda \phi_{\text {end }}^{4} / 12$, the above inequality can be cast into the following form

$$
\frac{3}{2 \sqrt{\pi}} \frac{m_{\mathrm{Pl}}}{\phi_{\text {end }}}<\hat{k}<\left(\frac{3 \sqrt{3}}{2 \pi}\right)^{1 / 2} \frac{m_{\mathrm{Pl}}}{\phi_{\text {end }}} .
$$

For the particular case of quartic large field inflation, $\phi_{\text {end }} / m_{\mathrm{Pl}} \simeq 1 / \sqrt{\pi}$, hence the lower and upper bound of the above band reduce respectively to $3 / 2$ and $(3 \sqrt{3} / 2)^{1 / 2}$.

For the above instability, the maximum growth occurs with a Floquet index $\mu \simeq 0.036$ at $k^{2} /\left(\phi_{\text {end }}^{2} a_{\text {end }}^{2} \lambda\right) \simeq$ 1.615 (i.e. $\hat{k} \simeq 1.56$ for a large field quartic inflationary model) [5]. The Floquet index is constant, therefore growth becomes exponential, unlike what has been found for the quadratic case.

Several remarks are in order at this point. Firstly, the resonance band corresponds to a fixed narrow range of comoving scales, contrary to the quadratic case for which the band widens with time. Secondly, the modes that are subject to parametric resonance are of spatial scale comparable with the Hubble radius at the end of inflation. Thirdly, the effect found here is similar to that investigated in Ref. [4, 5] except that it concerns Mukhanov's variable rather than the perturbed inflaton field or the Fourier amplitude of a scalar field $\chi$ coupled to the inflaton as $g^{2} \phi^{2} \chi^{2} / 2$, where $g$ is the coupling constant. The case of density perturbations can be viewed as a special case with a specific value of $g$. But, of course, the underlying physics is different in the sense that metric fluctuations are different from an external scalar field coupled to $\phi$.

In order to check the effect discussed above, we have numerically integrated the equation of motion for a large field inflationary quartic potential, see Fig. 6. The numerical analysis confirms the previous analytical analysis, although we find that the instability band is shifted by $12 \%$ to higher values in comparison with Eq. (47); this effect is likely due to our assumption of instantaneous transition from inflation to a (radiation dominated) preheating era in the analytical calculations. In detail, one finds the band at $1.684<\hat{k}<1.807$. When the mode is outside the resonance band (left panel in Fig. 6), the perturbations remain constant while when it is inside (right panel in Fig. (6), we observe a violent amplification of $\Phi_{\boldsymbol{k}}$, $\zeta_{\boldsymbol{k}}$ and $\delta_{\boldsymbol{k}}$.

To summarize, the quartic case presents substantial differences in comparison with the quadratic case. There 

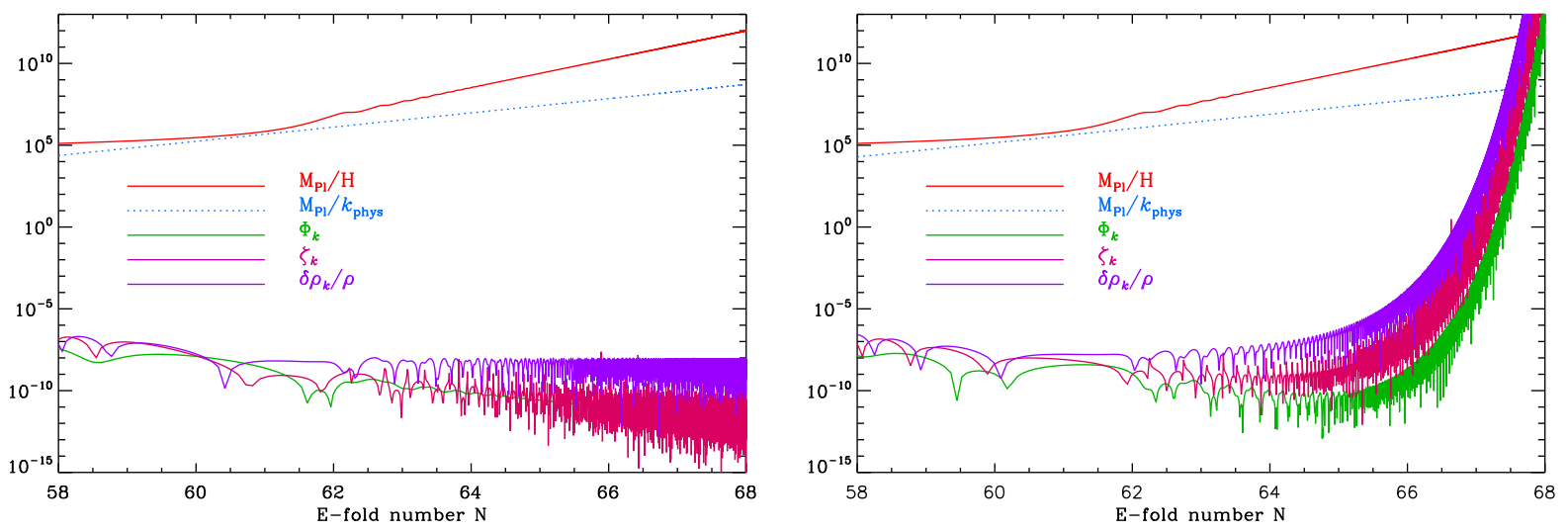

FIG. 6: Evolution of the density perturbations $\delta \rho_{\boldsymbol{k}} / \rho, \zeta_{\boldsymbol{k}}$ and $\Phi_{\boldsymbol{k}}$ towards the end of quartic inflation and during the ensuing period of metric preheating after inflation. The dimensionless parameter is $\lambda \simeq 10^{-12}$. The red solid line represents the Hubble radius while the blue dotted line represents the physical wavelength of the Fourier mode. Left panel: the Fourier mode is characterized by $\hat{k} \simeq 1.427$ and lies outside the resonance band. Right panel: $\hat{k} \simeq 1.738$ and the mode is inside the instability band as the exponential growth indicates.

exists a narrow instability band at scales of order of the Hubble radius at the end of inflation, of fixed width (in terms of comoving wavenumber) and with associated exponential growth.

\section{B. The mixed quadratic-quartic case}

The purely quartic case arises only if the quadratic term is absent in the potential. Here we briefly address the more general case, in which both quadratic and quartic terms are present:

$$
V(\phi)=\frac{1}{2} m^{2} \phi^{2}+\frac{\lambda}{4} \phi^{4} .
$$

At large vacuum expectation values of the inflaton, the quartic term always dominates while at small values of $\phi$, the quadratic term is the most important one. For which value of $\phi$ these terms are of equal importance depends on the two free parameters $m$ and $\lambda$. If the potential were purely quartic, then inflation would stops at $\phi_{\text {end }}=$ $m_{\mathrm{Pl}} / \sqrt{\pi}$. For this value, the two terms are equal if $m=$ $\sqrt{\lambda /(2 \pi)} m_{\mathrm{Pl}}$. It is therefore convenient to parameterize the potential (48) by $\lambda$ and the dimensionless parameter $\Upsilon$ defined by

$$
\Upsilon \equiv \frac{m}{m_{\mathrm{Pl}}} \sqrt{\frac{2 \pi}{\lambda}} .
$$

The regime where $\Upsilon \gg 1$ reduces to the quadratic case while the case $\Upsilon \ll 1$ is equivalent to the quartic case. The most interesting situation is of course when $\Upsilon$ is of order one.

In order to study this case, we have numerically integrated the equations of motion, assuming that the potential form remains the same during inflation and the preheating era. As a first example, we have taken $\lambda=10^{-12}$, that is to say the same value as in the previous section for numerical applications and $\Upsilon=0.05$. We have also chosen $\hat{k}=1.738$, i.e. a value which is inside the resonance band in the pure quartic case, see right panel in Fig. 6] The result is displayed in Fig. 7 (left panel). Several comments are in order. Firstly, one notices that the Hubble radius and the characteristic scale $\ell_{\mathrm{C}}$ no longer coincide. This is of course due to the fact that the mass $m$ is no longer COBE normalized. In fact, in this model, the end of inflation is delayed because at $\phi=m_{\mathrm{Pl}} / \sqrt{\pi}$, the quadratic term still plays a role. Regarding the perturbations themselves, despite the fact that the Fourier mode is inside the quartic resonance band of Eq. (47), there is no amplification. It is of course due to the fact that, for small vacuum expectation values of the field, the dominant term is the quadratic one. We also notice that $\delta_{\mathbf{k}}$ does not grow as $a$ either. This is because, since the end of inflation has been postponed and the value of $\ell_{\mathrm{C}}$ increased (in comparison with the corresponding value in the pure quadratic case), the time at which the mode crosses $\ell_{\mathrm{C}}$ is also delayed (and therefore lies outside of the plot).

It is clear that if we decrease the value of $\Upsilon$, and keep the same value for $k$, we should observe the growth described in the last section in the pure quartic case. This is indeed the case as demonstrated in Fig. 7 (right panel) where $\Upsilon=0.003$. Just after the end of inflation, the Fourier mode is inside the pure quartic resonance band and the perturbations grow. But as the amplitude of the inflaton field decreases, the influence of the quadratic term becomes more and more important. As a consequence, the exponential growth stops at some point. Then, the amplitude of the fluctuations remain constant until the mode exceeds the characteristic scale $\ell_{\mathrm{C}}$ associated to the quadratic part of the potential. As before, this time is delayed due to the small value of the mass $m$ 

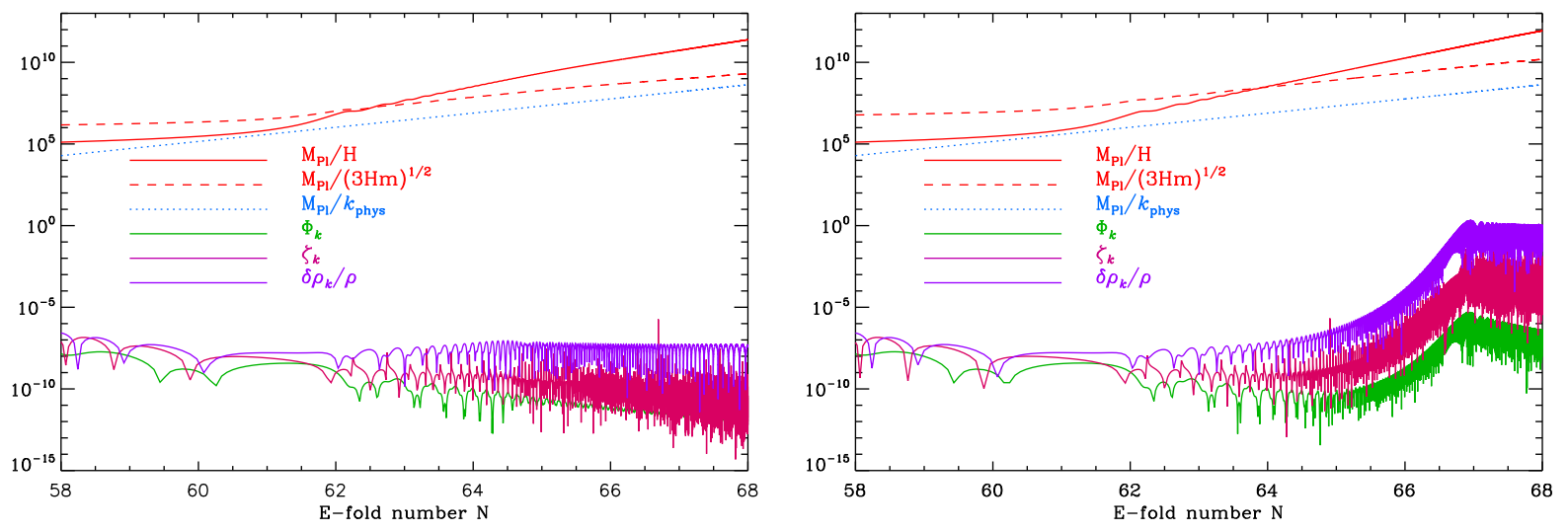

FIG. 7: Left panel: evolution of the density perturbations $\delta \rho_{\boldsymbol{k}} / \rho, \zeta_{\boldsymbol{k}}$ and $\Phi_{\boldsymbol{k}}$ towards the end of inflation with a potential given by Eq. (48) and during the ensuing period of of metric preheating. The dimensionless parameter are $\lambda \simeq 10^{-12}$ and $\Upsilon=0.05$. The red solid line represents the Hubble radius, the red dashed line the scale $\ell_{\mathrm{C}}$ while the blue dotted line represents the physical wavelength of the Fourier mode. The Fourier mode is characterized by $\hat{k} \simeq 1.738$. Right panel: same as left panel but with $\Upsilon=0.003$ and $\hat{k}=1.737$. The value of $k$ is the same for the two panels but the value of $\hat{k}$ sightly differs because the end of inflation does not occur exactly at the same time in the two cases.

(small in comparison with the COBE normalized value) and lies outside of the plot. Then, the density contrast grows proportional to the scale factor as discussed in Section III.

\section{CONCLUSION}

In this paper we have investigated the evolution of subhorizon perturbations between the end of cosmic inflation and the beginning of a radiation dominated early Universe. This study assumes that energy density of the Universe during this phase is dominated by the oscillations of a single scalar field around its minimum in a quadratic or quartic potential.

We have found that perturbations are subject to a preheating instability in the narrow resonance regime, leading to the growth of the density contrast $\delta \rho_{\boldsymbol{k}} / \rho$ on sub-horizon scales. In the case of a quadratic potential, the domain of instability (in terms of comoving wavenumbers) widens with time. This implies that a wavenumber that has entered the band at some time remains inside for the whole duration of the preheating era. The density contrast of sub-horizon unstable modes then increases as the scale factor, reaching nonlinearity for a wide range of scales. Maximum growth occurs for spatial scales of the order of the Hubble radius at the end of inflation, to which is associated a mass scale $M_{\text {end }} \simeq 3.3 \times 10^{3} \mathrm{~g}\left(H_{\mathrm{end}} / 10^{13} \mathrm{GeV}\right)^{-1}$.
A quartic potential leads to a different phenomenology, although the main effect of preheating instability remains. Notably, the range of unstable wavenumbers is now fixed in terms of comoving wavenumbers and peaked around the Hubble scale at the end of inflation. On the other hand, the density contrast of sub-horizon unstable modes increases exponentially fast.

The growth of structure on sub-horizon scales is known to occur in a matter dominated era, hence the growth of the density contrast for a quadratic inflaton potential can thus be well understood in this respect. However, the result obtained for a quartic potential confirms that the effect goes beyond a simple analogy with Newtonian collapse of a self-gravitating dust cloud, as the effective equation of state in this latter case is that of radiation.

These results open interesting avenues of research in the search for observational signatures of the dynamics of the very early Universe. In particular, as we have noted, the abundance of black holes of mass comparable to the above is constrained by cosmological arguments. The formation of inflaton halos may also lead to copious production of gravitational waves, as discussed in a companion paper. Finally, the very existence of nonlinearities on small scales casts some shadow on the reliability of the predictions for curvature perturbations on those large scales responsible for the present large-scale structure. These latter could be modified if effects of mode-mode coupling become important.
[1] A. D. Linde, Phys. Lett. B129, 177 (1983).

[2] A. D. Linde, Lect. Notes Phys. 738, 1 (2008), 0705.0164.
[3] E. Komatsu et al., (2010), 1001.4538.

[4] L. Kofman, A. D. Linde, and A. A. Starobinsky, Phys. 
Rev. D56, 3258 (1997), hep-ph/9704452.

[5] B. A. Bassett, S. Tsujikawa, and D. Wands, Rev. Mod. Phys. 78, 537 (2006), astro-ph/0507632.

[6] J. R. Ellis, A. D. Linde, and D. V. Nanopoulos, Phys. Lett. B118, 59 (1982).

[7] D. V. Nanopoulos, K. A. Olive, and M. Srednicki, Phys. Lett. B127, 30 (1983).

[8] K. Jedamzik, Phys. Rev. D74, 103509 (2006), hep$\mathrm{ph} / 0604251$.

[9] M. Kawasaki, K. Kohri, T. Moroi, and A. Yotsuyanagi, Phys. Rev. D78, 065011 (2008), 0804.3745.

[10] S. Bailly, K.-Y. Choi, K. Jedamzik, and L. Roszkowski, JHEP 05, 103 (2009), 0903.3974.

[11] Y. Nambu and A. Taruya, Prog. Theor. Phys. 97, 83 (1997), gr-qc/9609029.

[12] T. Hamazaki and H. Kodama, Prog. Theor. Phys. 96, 1123 (1996), gr-qc/9609036.

[13] F. Finelli and R. H. Brandenberger, Phys. Rev. Lett. 82, 1362 (1999), hep-ph/9809490.

[14] M. Khlopov, B. A. Malomed, and I. B. Zeldovich, Mon. Not. Roy. Astron. Soc. 215, 575 (1985).

[15] Y. Nambu and M. Sasaki, Phys. Rev. D42, 3918 (1990).

[16] B. Ratra, Phys. Rev. D44, 352 (1991).

[17] J.-C. Hwang, Phys. Lett. B401, 241 (1997), astro$\mathrm{ph} / 9610042$.

[18] V. F. Mukhanov, H. A. Feldman, and R. H. Brandenberger, Phys. Rept. 215, 203 (1992).

[19] J. Martin, Braz. J. Phys. 34, 1307 (2004), astro$\mathrm{ph} / 0312492$.

[20] J. Martin, Lect. Notes Phys. 669, 199 (2005), hepth/0406011.

[21] J. Martin, Lect. Notes Phys. 738, 193 (2008), 0704.3540.

[22] J. Martin and D. J. Schwarz, Phys. Rev. D57, 3302 (1998), gr-qc/9704049.

[23] H. Kodama and T. Hamazaki, Prog. Theor. Phys. 96, 949 (1996), gr-qc/9608022.

[24] J. Martin and C. Ringeval, JCAP 0608, 009 (2006), astro-ph/0605367.
[25] L. Lorenz, J. Martin, and C. Ringeval, JCAP 0804, 001 (2008), 0709.3758.

[26] L. Lorenz, J. Martin, and C. Ringeval, Phys. Rev. D78, 063543 (2008), 0807.2414

[27] S. M. Leach, A. R. Liddle, J. Martin, and D. J. Schwarz, Phys. Rev. D66, 023515 (2002), astro-ph/0202094.

[28] D. J. Schwarz, C. A. Terrero-Escalante, and A. A. Garcia, Phys. Lett. B517, 243 (2001), astro-ph/0106020.

[29] J.-O. Gong and E. D. Stewart, Phys. Lett. B510, 1 (2001), astro-ph/0101225.

[30] J. Martin and D. J. Schwarz, Phys. Rev. D67, 083512 (2003), astro-ph/0210090.

[31] J. M. Bardeen, Phys. Rev. D22, 1882 (1980).

[32] J. Lesgourgues, D. Polarski, and A. A. Starobinsky, Nucl. Phys. B497, 479 (1997), gr-qc/9611019.

[33] A. M. Green, Phys. Rev. D60, 063516 (1999), astro$\mathrm{ph} / 9903484$.

[34] M. Lemoine, Phys. Lett. B481, 333 (2000), hep$\mathrm{ph} / 0001238$.

[35] M. Y. Khlopov, A. Barrau, and J. Grain, Class. Quant. Grav. 23, 1875 (2006), astro-ph/0406621.

[36] B. J. Carr, J. H. Gilbert, and J. E. Lidsey, Phys. Rev. D50, 4853 (1994), astro-ph/9405027.

[37] B. Carr, K. Kohri, Y. Sendouda, and J. Yokoyama, (2009), 0912.5297.

[38] L. P. Grishchuk, Sov. Phys. JETP 40, 409 (1975).

[39] D. I. Kaiser, Phys. Rev. D56, 706 (1997), hep$\mathrm{ph} / 9702244$.

[40] D. I. Kaiser, Phys. Rev. D57, 702 (1998), hep$\mathrm{ph} / 9707516$.

[41] M. Abramowitz and I. A. Stegun, Handbook of mathematical functions with formulas, graphs, and mathematical tables, ninth ed. (National Bureau of Standards, Washington, US, 1970).

[42] I. S. Gradshteyn and I. M. Ryzhik, Table of Integrals, Series, and Products (Academic Press, New York and London, 1965). 